\title{
Does preoperative atrial fibrillation influence early and late outcomes of coronary artery bypass grafting?
}

Dumbor L. Ngaage, MB, BS, ${ }^{a}$ Hartzell V. Schaff, MD, ${ }^{a}$ Charles J. Mullany, MB, MS, ${ }^{\text {a }}$ Thoralf M. Sundt III, MD, ${ }^{a}$ Joseph A. Dearani, MD, ${ }^{a}$ Sunni Barnes, PhD, ${ }^{b}$ Richard C. Daly, MD, and Thomas A. Orszulak, MDa

From the Division of Cardiovascular Surgery $^{\mathrm{a}}$ and Department of Biostatistics, Mayo Clinic College of Medicine, Rochester, Minn.

Received for publication June 6, 2006; revisions received Aug 1, 2006; accepted for publication Sept 7, 2006.

Reprint requests: Dumbor L. Ngaage, MB, BS, Department of Cardiothoracic Surgery, Cardiothoracic Centre, Castle Hill Hospital, Cottingham, East Yorkshire HU16 5JQ, United Kingdom (E-mail:dngaage@yahoo.com).

J Thorac Cardiovasc Surg 2007;133:182-9

$0022-5223 / \$ 32.00$

Copyright () 2007 by The American Association for Thoracic Surgery

doi:10.1016/j.jtcvs.2006.09.021
Objective: The study objective was to describe the independent effect of preoperative atrial fibrillation on the outcome of coronary artery bypass grafting, including the causes of death (cardiac vs noncardiac).

Methods: We analyzed the outcome of patients with preoperative atrial fibrillation who underwent on-pump coronary artery bypass grafting between 1993 and 2002 and compared them with matched controls in sinus rhythm; matching variables were age, gender, ejection fraction, and numbers of diseased coronary arteries and distal anastomoses. Direct patient follow-up focused on late complications and reinterventions, and we investigated causes for all deaths.

Results: Operative mortality ( $1.6 \%$ vs $1.9 \%, P=.79)$ was similar in patients with preoperative atrial fibrillation $(\mathrm{n}=257)$ compared with patients in sinus rhythm $(\mathrm{n}$ $=269)$. The patients with atrial fibrillation had longer hospital stays $(9 \pm 6$ days vs $8 \pm 6$ days, $P=.0008)$ and a trend to more frequent early readmissions (13\% vs $9 \%, P=.08$ ). During follow-up (median 6.7 years, maximum 12 years), late hospital admission was more frequent in patients with atrial fibrillation (59\% vs $31 \%, P<.0001$ ). Risk of late mortality (all causes) in patients with atrial fibrillation was increased by $40 \%$ compared with patients in sinus rhythm $(P=0.02)$, and the late cardiac death rate in the atrial fibrillation group was 2.8 times that of the sinus rhythm group $(P=.0004)$. Major adverse cardiac events occurred in $70 \%$ of patients with preoperative atrial fibrillation compared with $52 \%$ of patients in preoperative sinus rhythm $(P<.0001)$. Subsequent rhythm-related intervention, including pacemaker implantations, was more common in the atrial fibrillation group (relative risk $=2.1, P=.0027$ ).

Conclusions: Uncorrected preoperative atrial fibrillation in patients undergoing coronary artery bypass grafting is associated with increased late cardiac morbidity and mortality and poor long-term survival. These data support consideration of atrial fibrillation surgery at the time of coronary artery bypass grafting.

A trial fibrillation (AF) is a common tachyarrhythmia, affecting approximately 2.2 million people in the United States, and the median age of patients with $\mathrm{AF}$ is 70 years. ${ }^{1}$ As a result of the increasing age of patients requiring myocardial revascularization and the predisposing influence of ischemic heart disease, $\mathrm{AF}$ is not infrequent in patients undergoing coronary artery bypass grafting (CABG). Several population-based studies and nonsurgical reports ${ }^{2-4}$ have identified $\mathrm{AF}$ as a marker of increased cardiovascular morbidity and mortality because of its association with other cardiovascular diseases. There are few studies examining the impact of concurrent AF on the long-term outcome in patients after surgical correction of the associated cardiovascular disease.

Quader and associates ${ }^{5}$ reported a reduction in long-term survival in patients undergoing $\mathrm{CABG}$ with preoperative $\mathrm{AF}$ and recommended concomitant surgical 


$$
\begin{aligned}
& \text { Abbreviations and Acronyms } \\
& \begin{aligned}
\mathrm{AF} & =\text { atrial fibrillation } \\
\mathrm{CABG} & =\text { coronary artery bypass grafting } \\
\mathrm{CI} & =\text { confidence interval } \\
\mathrm{SR} & =\text { sinus rhythm } \\
\mathrm{RR} & =\text { relative risk }
\end{aligned}
\end{aligned}
$$

ablation. Apart from this study, which focused only on overall survival, there are no studies that comprehensively evaluate the long-term adverse effects of uncorrected preoperative $\mathrm{AF}$ after $\mathrm{CABG}$ in patients receiving optimal medical treatment. Moreover, the impact of the clinical type and duration of $\mathrm{AF}$ on outcome in this patient population is not known. Because of current enthusiasm for surgical ablation of $\mathrm{AF}$, it is important to investigate the influence of $\mathrm{AF}$ on outcome to identify which patients might benefit from concomitant ablation procedures.

The objectives of this study were to determine the prevalence of AF in patients undergoing CABG and to compare the early and late clinical outcome between patients with preoperative $\mathrm{AF}$ and patients in normal sinus rhythm (SR), with specific reference to adverse cardiac and cerebrovascular events and long-term survival; further, the study addressed the influence of type and duration of AF on clinical outcome.

\section{Patients and Methods \\ Study Population and Matching}

The cardiovascular surgery database of our institution contains information on 7498 patients who underwent isolated CABG between January 1993 and December 2002, of whom 650 (8.7\%) had preoperative AF. The institutional review board approved the use of patients' data for this study. Patients were excluded if they had a history of cardiac arrhythmia other than AF, permanent sequelae of stroke, prior implantation of a permanent pacemaker or cardioverter defibrillator, prior AF ablation, surgery within 7 days of acute myocardial infarction, and off-pump CABG. Patients with cancer who had a limited life expectancy were also excluded. Of 4151 patients who met the study criteria and for whom complete matching variables were available, 284 had preoperative AF confirmed by electrocardiograms, and 3867 were in SR.

Previous publications suggested a $10 \%$ reduction in late survival of patients with AF undergoing $\mathrm{CABG}$ compared with controls; with these data, we estimated that 200 patients would be needed in each group to demonstrate this difference ( $90 \%$ power). We then used the Greedy method ${ }^{6}$ to identify the best matched control (preoperative SR) for each case based on age, gender, ejection fraction, extent of coronary artery disease, and the number of distal anastomoses. Demographic characteristics, clinical details, and perioperative variables from our database were supplemented by review of patient charts, operative notes, and electrocardiographic, angiographic, and echocardiographic reports (Table 1).

\section{Definitions}

Terminologies used in the description of the types of AF in the literature can be confusing. The international consensus on nomenclature and classification recently released a position paper on the clinical classification of $\mathrm{AF}^{7}$ which classifies $\mathrm{AF}$ into initial event, paroxysmal, persistent, and permanent types. During the study period this classification was not in use, and data relating to $\mathrm{AF}$ were recorded using the simpler classification proposed by $\mathrm{Cox}^{8}$ which divides $\mathrm{AF}$ on the basis of clinical presentation into intermittent and continuous types. However, both classifications share common arrhythmia patterns; paroxysmal and persistent $\mathrm{AF}$ are characterized by recurrent arrhythmia (eg, intermittent AF), and permanent $\mathrm{AF}$ (eg, continuous AF) has established arrhythmia. We therefore categorized preoperative $\mathrm{AF}$ on the basis of clinical presentation into paroxysmal/persistent and permanent types, and into short and long duration to enable comparison of clinical outcomes.

- Paroxysmal/persistent AF describes recurrent AF.

- Permanent AF denotes established AF that is present at all times.

- Short-duration AF refers to preoperative AF of 3 months' duration or less.

- Long-duration AF refers to preoperative AF of more than 3 months' duration.

\section{Follow-up}

Both the Social Security Death Index and Mayo Clinic's patient registration were queried for deaths, and certificates were obtained for all deaths. A survey questionnaire was then sent to all surviving patients in the study to gather information including symptom change, occurrence of adverse cardiac and cerebrovascular events, cardiac-related hospital readmission after the index CABG operation, further cardiac interventions, medications, and cardiac rhythm (Table 2).

Follow-up data were available for 257 patients with preoperative AF and 269 matched patients in SR. These 526 patients constitute the study population.

\section{Statistical Analysis}

The primary end points were (1) mortality, both overall mortality and cardiac-related deaths, and (2) major adverse cardiac and cerebrovascular events, which include cardiac death, myocardial infarction, heart failure, further cardiac intervention, and stroke. Secondary end points of this study were length of hospital stay, cardiac-related hospital readmissions, Coumadin and antiarrhythmic drug use, and bleeding/thromboembolic complications.

Categoric variables are expressed as percentages, and continuous variables are expressed as the mean \pm standard deviation, unless otherwise stated. Univariate analysis was performed using the chi-square test for qualitative data, the Wilcoxon rank test for quantitative data, and the Kaplan-Meier method for long-term outcomes. Multivariable analysis was done using Cox proportional hazards models and logistic regression. The stepwise model selection procedure was used to identify risk factors associated with each end point of interest. If the primary variable of interest (AF vs SR) was selected by the stepwise procedure, then this is the model reported in the results section. Otherwise, if the AF/SR grouping variable was not chosen, then it was forced into the final 
TABLE 1. Baseline characteristics and in-hospital outcome of patients undergoing coronary artery bypass grafting with and without preoperative atrial fibrillation

\begin{tabular}{|c|c|c|c|}
\hline Variable & $\begin{array}{c}\text { SR group } \\
(\mathrm{n}=269) \\
\text { No. }(\%) \\
\end{array}$ & $\begin{array}{c}\text { AF group } \\
(\mathrm{n}=257) \\
\text { No. }(\%)\end{array}$ & $\begin{array}{c}P \\
\text { value } \\
\end{array}$ \\
\hline \multicolumn{4}{|l|}{ Demographics } \\
\hline Mean age $(y)^{*}$ & $70 \pm 8$ & $71 \pm 8$ & .26 \\
\hline Female gender* & $57(21)$ & $54(21)$ & .96 \\
\hline Mean BMI (kg/m²) & $28 \pm 5$ & $29 \pm 5$ & .59 \\
\hline \multicolumn{4}{|l|}{ Clinical symptoms } \\
\hline Angina class & & & .16 \\
\hline I & $17(6)$ & $24(9)$ & \\
\hline II & $27(10)$ & $14(5)$ & \\
\hline III & $128(48)$ & $122(47)$ & \\
\hline IV & $97(36)$ & $97(38)$ & \\
\hline NYHA class & & & .22 \\
\hline 1 & $11(4)$ & $7(3)$ & \\
\hline II & $25(9)$ & $13(5)$ & \\
\hline III & 132 (49) & $136(53)$ & \\
\hline IV & $101(38)$ & $101(39)$ & \\
\hline Unstable angina & $42(16)$ & $31(12)$ & .24 \\
\hline \multicolumn{4}{|l|}{ History } \\
\hline Stroke & $27(10)$ & $27(11)$ & .86 \\
\hline Carotid endarterectomy & $10(4)$ & $6(2)$ & .36 \\
\hline Angioplasty/stent & $70(26)$ & $85(33)$ & .08 \\
\hline \multicolumn{4}{|l|}{ Comorbidities } \\
\hline Diabetes mellitus & $69(26)$ & $89(35)$ & .02 \\
\hline Renal insufficiency & $13(5)$ & $14(5)$ & .75 \\
\hline Hypertension & $192(71)$ & $190(74)$ & .51 \\
\hline COPD & $25(9)$ & $36(14)$ & .09 \\
\hline \multicolumn{4}{|l|}{ Cardiac morbidity } \\
\hline Mean ejection fraction* & $0.56 \pm 0.16$ & $0.53 \pm 0.14$ & .14 \\
\hline Coronary artery disease* & & & .77 \\
\hline 1-vessel & $8(3)$ & $8(3)$ & \\
\hline 2-vessel & $42(16)$ & $46(18)$ & \\
\hline 3-vessel & $219(81)$ & $203(79)$ & \\
\hline Left main stem disease & $74(28)$ & $80(31)$ & .36 \\
\hline Myocardial infarction & $139(52)$ & $141(55)$ & .46 \\
\hline Cardiomegaly & $84(32)$ & $111(43)$ & .007 \\
\hline \multicolumn{4}{|l|}{ Operative details } \\
\hline Surgery priority & & & .32 \\
\hline Elective & $240(89)$ & 238 (93) & \\
\hline Urgent & $22(8)$ & $16(6)$ & \\
\hline Emergency & $7(3)$ & $3(1)$ & \\
\hline Distal anastomosis* & & & .96 \\
\hline 1 & $3(1)$ & $2(1)$ & \\
\hline 2 & 38 (14) & $30(12)$ & \\
\hline 3 & $115(43)$ & $114(44)$ & \\
\hline 4 & $93(35)$ & $93(36)$ & \\
\hline 5 & $19(7)$ & $17(7)$ & \\
\hline 6 & $1(0)$ & $1(0)$ & \\
\hline ITA use & & & .53 \\
\hline None & $11(4)$ & $15(6)$ & \\
\hline Left & 247 (92) & $233(91)$ & \\
\hline Right & $2(1)$ & 0 & \\
\hline
\end{tabular}

TABLE 1. (Continued)

\begin{tabular}{lccc}
\hline & $\begin{array}{c}\text { SR group } \\
\text { (n= 269) } \\
\text { No. (\%) }\end{array}$ & $\begin{array}{c}\text { AF group } \\
\text { (n = 257) } \\
\text { No. (\%) }\end{array}$ & $\begin{array}{c}\boldsymbol{P} \\
\text { value }\end{array}$ \\
Variable & $9(3)$ & $9(3)$ & \\
\hline Bilateral & $50 \pm 18$ & $50 \pm 18$ & .52 \\
Mean crossclamp & & & \\
$\quad$ time (min) & $92 \pm 32$ & $91 \pm 33$ & .44 \\
Perfusion time & & & \\
Postoperative data & $117(43)$ & $129(50)$ & .12 \\
$\quad$ Inotropic support & $17(6)$ & $11(4)$ & .30 \\
$\quad$ IABP use & $13(5)$ & $8(3)$ & .31 \\
$\quad$ Reoperation for bleeding & $2(1)$ & $1(0)$ & 1.00 \\
$\quad$ Sternal wound infection & $8(3)$ & $8(3)$ & .93 \\
$\quad$ Stroke & $20(7)$ & $26(10)$ & .28 \\
$\quad$ Prolonged ventilation & $15(6)$ & $17(7)$ & .62 \\
$\quad$ Renal failure & $5(2)$ & $4(2)$ & 1.00 \\
Operative death & $8.0 \pm 5.6$ & $9.3 \pm 6.3$ & .0008 \\
Hospital length of stay & $23(9)$ & $34(13)$ & .08 \\
30-d readmission &
\end{tabular}

$S R$, Sinus rhythm; $A F$, atrial fibrillation; $B M I$, body mass index; $N Y H A$, New York Heart Association; COPD, chronic obstructive pulmonary disease; ITA, internal thoracic artery; $I A B P$, intra-aortic balloon pump. *Matching variables.

model provided by the stepwise procedure. The SAS statistical analysis system (SAS Institute Inc, Cary, NC) was used for data analysis.

\section{Results}

In-hospital Outcome

Table 1 compares patient demographics and baseline variables. The 2 groups were similar aside from cardiomegaly and diabetes, which were more prevalent in the AF group.

The AF and SR groups did not differ in operative characteristics or rates of perioperative complications. The operative (30-day) mortality in the $\mathrm{AF}$ group was $1.6 \%$ versus $1.9 \%$ for the SR group $(P=.79)$. Patients with preoperative AF had a longer average length of postoperative hospital stay compared with patients in SR $(9 \pm 6$ days vs $8 \pm 6$ days, $P=.0008$ ).

\section{Late Outcome}

The median follow-up for the entire study population was 6.7 years (maximum 12 years). Both the AF and SR groups had an equivalent duration of follow-up (6.7 vs 6.6 years). Symptom improvement, as measured by change in the angina class $(P=.51)$, and New York Heart Association functional class $(P=.74)$ were comparable between the 2 groups. Patients with preoperative AF had a tendency toward more readmissions within 30 days postoperatively for problems including congestive heart failure, AF, recurrent angina, and cardiac procedures $(13 \%$ vs $9 \%, P=.08)$. Beyond 30 days, the hospital readmission rate was significantly increased in patients with preoperative AF (54\% vs 
TABLE 2. Late results in the two groups of patients

\begin{tabular}{|c|c|c|c|c|c|}
\hline & \multicolumn{2}{|c|}{ SR group } & \multicolumn{2}{|c|}{ AF group } & \multirow[b]{2}{*}{$P$ value } \\
\hline & Total No. & $\begin{array}{c}\text { Frequency }(\%) \text { or } \\
\text { mean } \pm \text { SD }\end{array}$ & Total no. & $\begin{array}{c}\text { Frequency }(\%) \text { or } \\
\text { mean } \pm \text { SD }\end{array}$ & \\
\hline Late all-cause mortality & 264 & $86(33)$ & 253 & $104(41)$ & .02 \\
\hline Clinical symptoms & 119 & & 95 & & \\
\hline Angina class & & & & & .55 \\
\hline 1 & & $108(91)$ & & $84(88)$ & \\
\hline II & & $9(8)$ & & $8(8)$ & \\
\hline III & & $1(1)$ & & $3(3)$ & \\
\hline IV & & $1(1)$ & & 0 & .44 \\
\hline \multicolumn{6}{|l|}{ Angina class change } \\
\hline Increase & & 0 & & $2(2)$ & \\
\hline No change & & $10(8)$ & & $8(8)$ & \\
\hline Decrease & & $109(92)$ & & $85(89)$ & \\
\hline \multicolumn{6}{|l|}{ Medications } \\
\hline Coumadin & 140 & $17(12)$ & 121 & $68(56)$ & $<.0001$ \\
\hline Beta-blocker & 140 & $92(66)$ & 114 & $74(65)$ & .89 \\
\hline ACE inhibitor & 139 & $64(46)$ & 115 & $57(50)$ & .58 \\
\hline Antiarrhythmics & 138 & $20(14)$ & 112 & $55(49)$ & $<.0001$ \\
\hline Hospital admissions & 143 & $45(32)$ & 133 & $78(59)$ & $<.0001$ \\
\hline Cardiac rhythm & 141 & & 122 & & $<.0001$ \\
\hline SR & & $115(82)$ & & $34(28)$ & \\
\hline $\mathrm{AF}$ & & $24(17)$ & & $81(66)$ & \\
\hline Paced rhythm & & $2(1)$ & & $7(6)$ & \\
\hline \multicolumn{6}{|l|}{ Adverse events } \\
\hline \multicolumn{6}{|l|}{ Cardiac events } \\
\hline Cardiac death & 264 & $16(6)$ & 253 & $38(15)$ & .0004 \\
\hline Myocardial infarction & 140 & $13(9)$ & 110 & $11(10)$ & .85 \\
\hline Heart failure & 140 & $8(6)$ & 121 & $26(21)$ & .0002 \\
\hline Reintervention & 140 & $25(18)$ & 124 & $41(33)$ & .003 \\
\hline Stroke & 142 & $14(10)$ & 119 & $17(14)$ & .27 \\
\hline Bleeding/thromboembolism & 140 & $17(12)$ & 114 & $24(21)$ & .05 \\
\hline
\end{tabular}

$S D$, Standard deviation; $A C E$, angiotensin-converting enzyme; $S R$, sinus rhythm; $A F$, atrial fibrillation.

$32 \%, P<.0001)$. Overall (all-causes) mortality (relative risk $[\mathrm{RR}]=1.4, P=.02)$ and late cardiac death $(\mathrm{RR}=2.8$, $P=.0004)$ were more likely in the AF group compared with the SR group. In general, more major and minor adverse cardiac events were observed in the AF group $(70 \%$ vs $52 \%, P<.0001)$. Late congestive heart failure $(21 \%$ vs $6 \%, P=.0002)$, cardiac rhythm-related intervention $(35 \%$ vs $18 \%, P=.0027)$, and bleeding/thromboembolism $(21 \%$ vs $12 \%, P=.05)$ occurred more often in patients with preoperative AF. There was, however, no difference in late incidences of myocardial infarctions and strokes (Table 2). As expected, patients with preoperative AF had a higher use of Coumadin $(56 \%$ vs $12 \%, P<.0001)$ and antiarrhythmics (49\% vs $14 \%, P<.0001)$.

Patients with preoperative AF had comparatively poor late overall survival and freedom from late cardiac death (Figure 1, $A$, $B$ ). The 1-, 5-, and 10-year overall survivals were $96 \%, 74 \%$, and $41 \%$, respectively, for the AF group compared with $97 \%, 83 \%$, and $58 \%$, respectively, for the SR group $(P=.02)$. The corre- sponding 1-, 5-, and 10-year freedom from cardiac death values were $98 \%, 88 \%$, and $74 \%$, respectively, for the AF group compared with $100 \%, 97 \%$, and $90 \%$, respectively, for the SR group $(P=.0004)$. Figure 2 displays the trend of subsequent cardiac rhythm-related intervention after the index CABG operation in both groups of patients. More patients with AF underwent subsequent cardiac rhythm-related intervention in the form of cardioversion or AF ablation, and in the long term, patients with preoperative AF had a 2-fold increase in the risk of subsequent cardiac rhythm-related intervention compared with patients in SR.

\section{Preoperative Atrial Fibrillation As a Predictor of Adverse Outcomes After Coronary Artery Bypass Grafting}

In the multivariable analysis, preoperative $\mathrm{AF}$ was an independent predictor of prolonged hospital stay $(P=.03)$, major adverse cardiac events (hazard ratio 2.51 , 95\% confidence interval [CI]: 1.65-3.84, $P<.0001)$, all-cause mortality (RR $1.34,95 \% \mathrm{CI}: 0.995-1.80, P=.05)$, and cardiac 

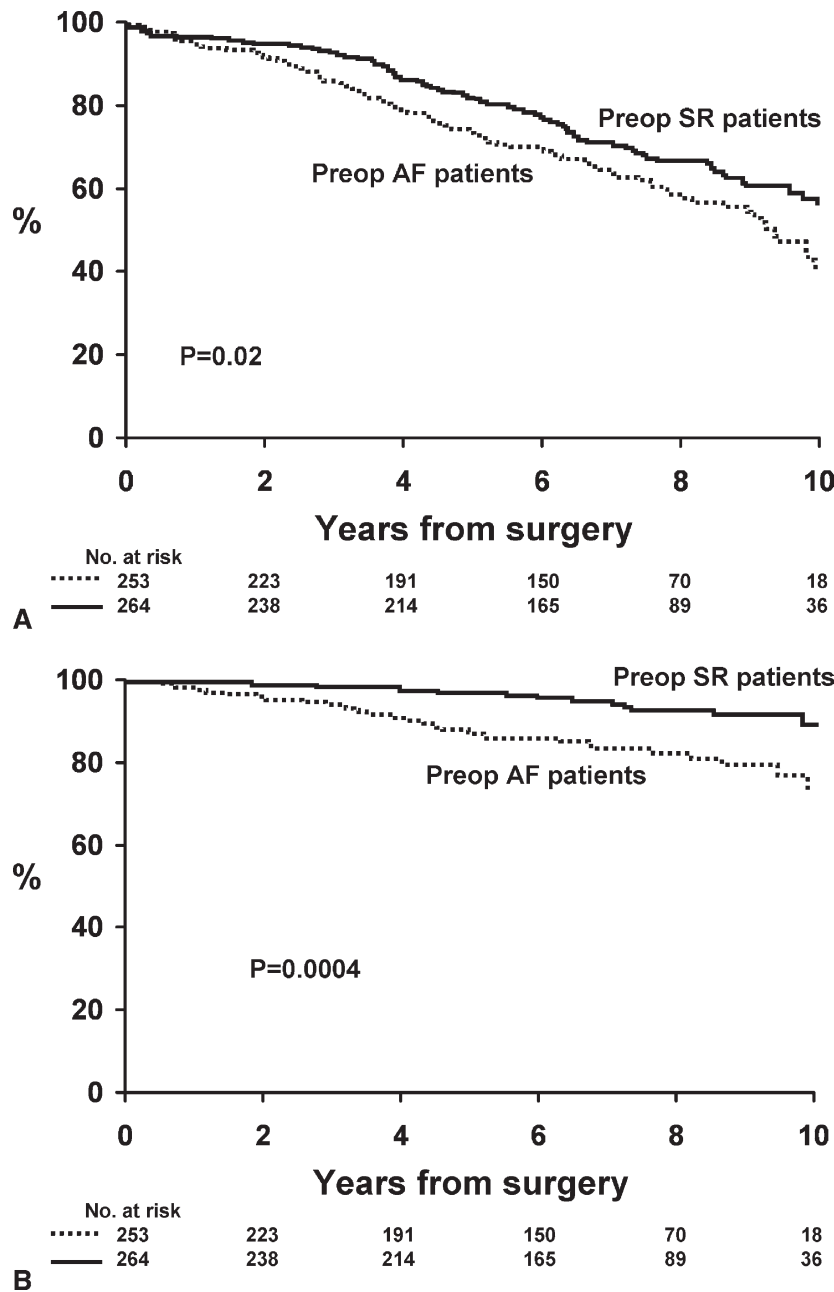

Figure 1. Kaplan-Meier survival curves for patients after CABG. A, Overall survival. B, Freedom from cardiac death. AF, Atrial fibrillation; $S R$, sinus rhythm.

death (hazard ratio $2.75,95 \% \mathrm{CI}$ : $1.50-5.02, P=.001$ ); this was true after controlling for other patient characteristics such as chronic obstructive pulmonary disease, diabetes, postoperative renal failure, cardiomegaly, congestive heart failure, preoperative stroke, and myocardial infarction. Table 3 shows other independent predictors of adverse outcome after $\mathrm{CABG}$.

Influence of Type and Duration of Atrial Fibrillation The type of preoperative AF was known with certainty in 225 patients $(88 \%)$. In this subset, late all-cause mortality was twice as likely to occur in patients with permanent preoperative AF compared with patients with paroxysmal/ persistent $\mathrm{AF}$ ( $\mathrm{RR}=2, P=.0007)$, resulting in 5- and 10 -year survivals of $67 \%$ and $22 \%$ vs $77 \%$ and $56 \%$, respectively (Figure 3, A). However, the rates of cardiac death

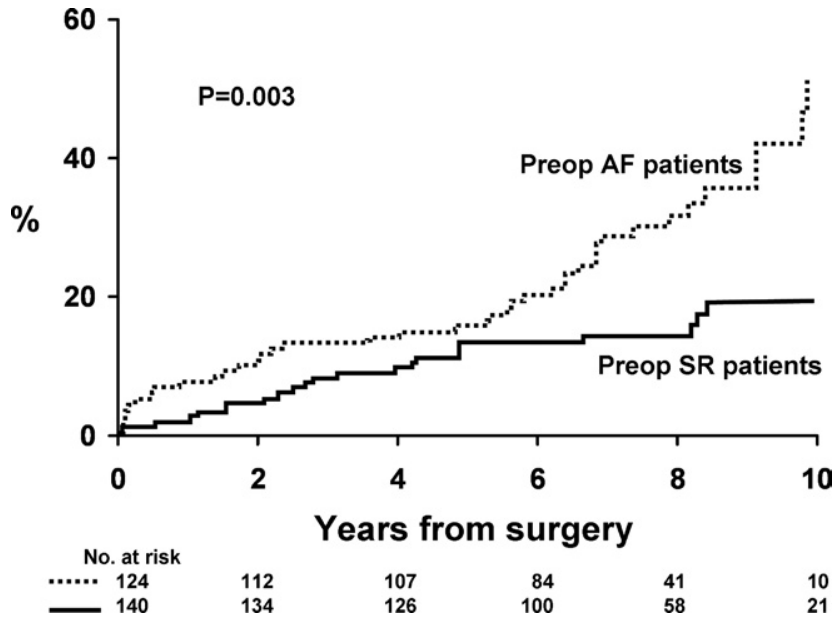

Figure 2. Trends for subsequent cardiac rhythm-related intervention after CABG in patients with preoperative $S R$ and $A F$. $A F$, Atrial fibrillation; $S R$, sinus rhythm.

and other adverse cardiac and cerebrovascular events were similar between patients with both types of AF. Among patients with known duration of AF (118 patients, 46\%), there was no difference in the length of hospital stay,

TABLE 3. Predictors of adverse clinical outcome by multivariate analysis

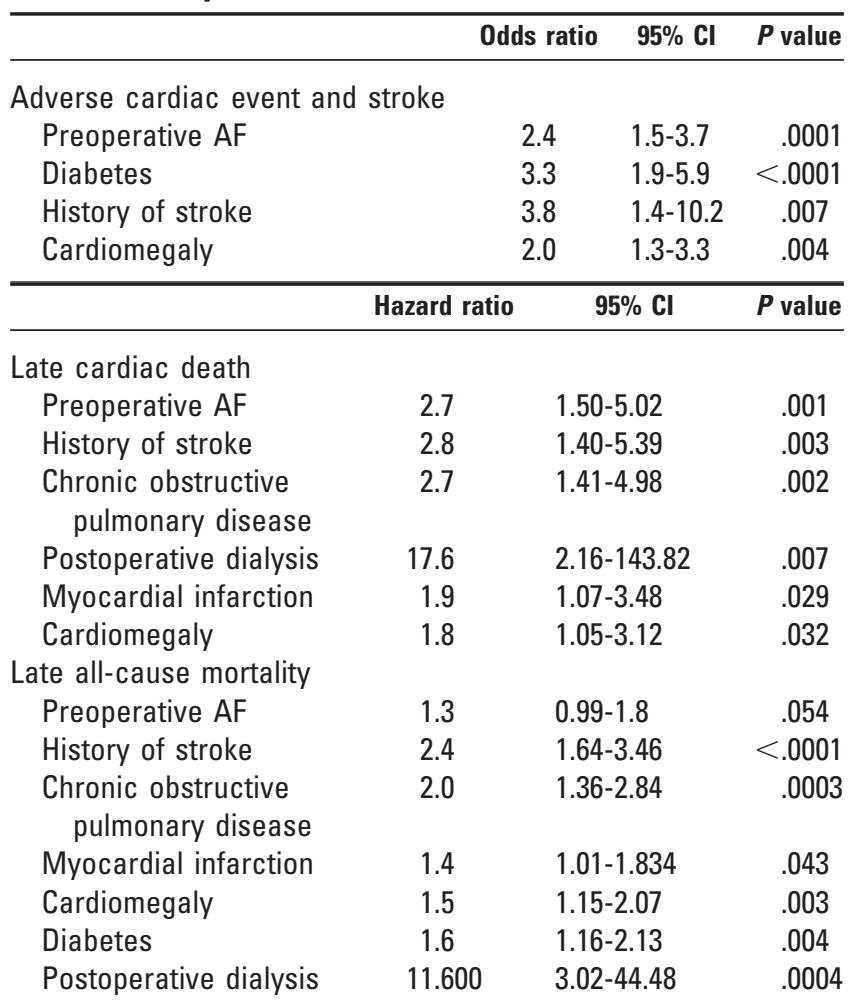

$\mathrm{Cl}$, Confidence interval; $A F$, atrial fibrillation. 

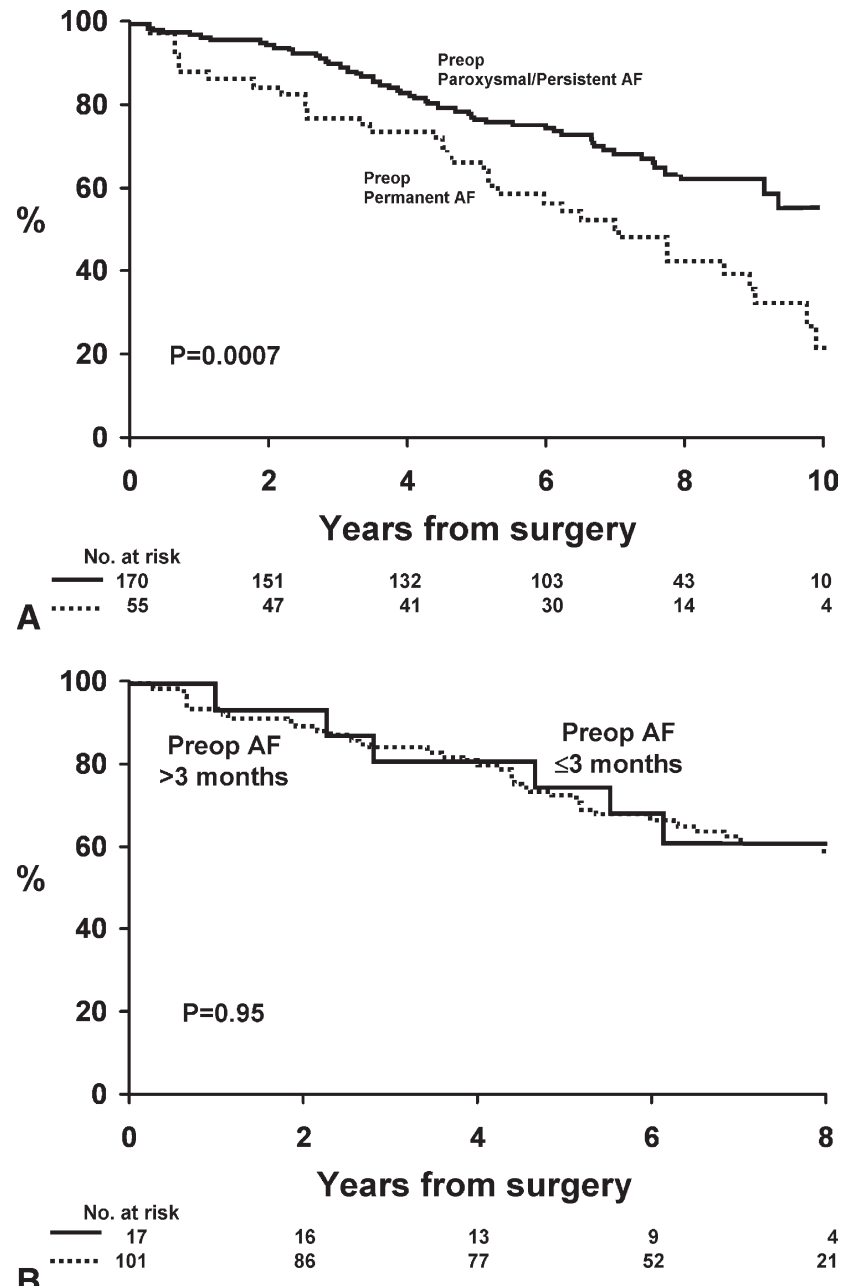

Figure 3. Kaplan-Meier survival curves for patients with different subclasses of preoperative AF after CABG. A, Survival of patients with the 2 patterns of AF. B, Comparison of survival on the basis of $A F$ duration. $A F$, Atrial fibrillation.

adverse cardiac and cerebrovascular events, and overall survival between patients with short-duration and longduration AF (Figure 3, B).

\section{Discussion}

The overall long-term survival of patients with preoperative $\mathrm{AF}$ undergoing $\mathrm{CABG}$ has been reported to be inferior to that of patients without preoperative AF. ${ }^{5}$ Quader and associates $^{5}$ reviewed 46,984 patients who underwent CABG between 1972 and 2000 and reported a preoperative AF prevalence rate of $0.96 \%$ (451 patients). They identified preoperative AF both as a marker of severe cardiovascular disease and as a determinant of poor long-term survival using propensity matching. Our patient population differs considerably as evidenced by the $8.7 \%$ prevalence of AF.
The present study also differs in that we conducted active follow-up and evaluated causes of late death and incidences of late morbid events, as well as the influence of types and duration of preoperative AF on clinical outcomes.

\section{Long-term Survival and Adverse Cardiac and Cerebrovascular Events}

Preoperative AF is associated with decreased long-term survival after CABG. New and notable findings from this study include increased rates of late adverse events, including death from cardiac causes such as heart failure and arrhythmia. Preoperative AF in patients undergoing CABG increased all-cause mortality by $40 \%$, the likelihood of cardiac death by approximately 3 folds $(\mathrm{RR}=2.8)$, and the risk of major adverse cardiac events by 2.5 times. The presence of preoperative AF doubled the risk of subsequent cardiac (rhythm-related) intervention, despite continuing optimal medical treatment of AF. The cohort of patients with AF in our study were receiving medical treatment with rate and/or rhythm control and anticoagulation, which may explain the relatively low rate of cerebrovascular accident in the AF group. Similarly, late myocardial infarction was rare in both groups. Notwithstanding, major cardiac adverse events (eg, heart failure and subsequent cardiac intervention) were more frequent in patients with preoperative AF. Impairment of both atrial and ventricular function occurs in $\mathrm{AF},{ }^{9,10}$ and this may contribute to the high rate of late postoperative congestive heart failure, which leads to clinical deterioration with poor prognosis. ${ }^{11}$ Together with recurrent episodes of rapid AF and recurrent angina, heart failure accounted for $86 \%$ of late hospital readmissions.

Many patients with AF are symptomatic with palpitations and fatigue, so it is not surprising that patients with $\mathrm{AF}$ were often readmitted for rate control and/or further rhythm-related intervention such as pacemaker placement with or without ablation of the atrioventricular node. Similar findings have been reported in nonsurgical series. ${ }^{12,13}$ Anticoagulation-related complications, such as gastrointestinal bleeding and epistaxis, occurred more often in patients with preoperative AF because of their high use of Coumadin. Both subtherapeutic and supratherapeutic levels of anticoagulation resulted in hospital readmission in some patients with AF in our study.

Beta-blockers and angiotensin-converting enzyme inhibitors confer a prognostic benefit in subsets of patients with coronary artery disease ${ }^{14,15}$ and after CABG. ${ }^{16}$ In this study, postoperative medical treatment was similar in patients with and without preoperative $\mathrm{AF}$ and thus does not explain the differences in late survival. Also, the use of antiarrhythmics for rate or rhythm control did not confer any survival advantage among patients with AF.

Among patients with preoperative AF, those with permanent AF were twice as likely to have late mortality. Gajewski and Singer ${ }^{17}$ first reported a differential survival 
impact of the clinical type of AF in a study of insurance applicants. Electrical and anatomic remodeling of the atria occurs in $\mathrm{AF}^{18,19}$ and leads to progression from recurrent to established arrhythmia ("AF begets AF"). It is important to note, however, that the duration of preoperative AF did not influence clinical outcomes after CABG. It is possible that the arbitrary 3-month cutoff used in this study and based on our previous report in patients with mitral valve disease ${ }^{20}$ may not be long enough in coronary artery disease for the development of sufficient morphologic changes to influence outcome.

The preoperative cardiac rhythm of patients did not affect late symptom improvement, probably because of the effect of CABG in relieving ischemia. Further, adequate medical treatment of AF can improve symptoms without affecting the long-term morbidity and mortality. ${ }^{21}$

Preexisting AF exerts a profound impact on prognosis after $\mathrm{CABG}$, but it remains to be determined whether $\mathrm{AF}$ ablation at the time of $\mathrm{CABG}$ would alter long-term results. Preoperative AF is a risk factor for adverse cardiac events but a weak predictor of all-cause mortality; frequently coexists with cardiomegaly, which has similar effects on clinical outcome (Table 3). Thus, it is possible that the correction of AF could achieve greater clinical benefit in patients without cardiomegaly. However, even in patients with cardiomegaly and history of stroke, the likelihood of poor outcome may also be reduced by AF ablation. These data also support consideration of AF surgery in patients with permanent AF.

\section{Strengths and Limitations}

This study was designed to compare the clinical outcomes of matched groups of patients who had preoperative AF with those in preoperative SR and was adequately powered to detect important differences in the primary and secondary dependent variables. The comprehensive investigation of the extent of the impact of preoperative $\mathrm{AF}$ on the late clinical outcome in patients managed with optimal medical treatment is relevant to contemporary practice and therefore a valuable addition to the cardiovascular medicine literature.

Although the groups were matched for important prognostic variables, more patients with preoperative AF had diabetes and cardiomegaly, which can affect outcome. To control the influence of these variables, we used the stepwise model selection procedure in the multivariate analysis. In the subset analysis of the impact of AF types, we combined paroxysmal and persistent AF on the basis of a similar arrhythmia pattern, because the distinction between the 2 can be subtle and difficult to reliably establish in a retrospective study. This categorization is consistent with current medical and surgical therapeutic strategies of managing AF. Another potential weakness of this study is recall bias due to reliance on self-reporting for follow-up data acquisition. The influence of this limitation is reduced by the supplemental follow-up data collection from hospital records for patients who attended the Mayo Clinic integrated heath care system. Moreover, the follow-up information obtained related to major events that patients are more likely to recall. Follow-up heart rhythm was confirmed by electrocardiography in only $15 \%$ of patients who were under surveillance locally; otherwise, the patients' responses to the questionnaire were accepted. It is noteworthy that in the patients with follow-up electrocardiograms, there was 100\% concordance with the questionnaire response for the late cardiac rhythm.

\section{Conclusions}

Preoperative AF in patients undergoing on-pump CABG predisposes them to high rates of early and late cardiac morbidity. Hospital stay after surgery is prolonged, and late hospital readmissions for cardiac-related events are common. Patients with preexisting AF have adverse cardiac events more frequently than those in normal SR after CABG despite optimal postoperative medical treatment with rate and/or rhythm control and anticoagulation. Long-term survival in patients with preoperative AF is steadily reduced by higher rates of cardiac and all-cause mortality. Patients with permanent AF have worse long-term survival compared with patients with paroxysmal/persistent AF. Whether ablation of $\mathrm{AF}$ at the time of $\mathrm{CABG}$ would improve the prognosis is yet to be determined, but these data support consideration of concomitant AF surgery.

\section{References}

1. Feinberg WM, Blackshear JL, Laupacis A, Kronmal R, Hart RG. Prevalence, age distribution, and gender of patients with atrial fibrillation. Analysis and implications. Arch Intern Med. 1995;155:469-73.

2. Benjamin EJ, Wolf PA, D'Agostino RB, Silbershatz H, Kannel WB, Levy D. Impact of atrial fibrillation on the risk of death: the Framingham Heart Study. Circulation. 1998;98:946-52.

3. Stewart S, Hart CL, Hole DJ, McMurray JJ. A population-based study of the long-term risks associated with atrial fibrillation: 20-year follow-up of the Renfrew/Paisley study. Am J Med. 2002;113:359-64.

4. Vidaillet H, Granada JF, Chyou PH, et al. A population-based study of mortality among patients with atrial fibrillation or flutter. Am J Med. 2002;113:365-70.

5. Quader MA, McCarthy PM, Gillinov AM, et al. Does preoperative atrial fibrillation reduce survival after coronary artery bypass grafting? Ann Thorac Surg. 2004;77:1514-22; discussion 22-4.

6. Aronson J, Dyer M, Frieze A, Suen S. Randomized greedy matching. 2. Random Struct Algor. 1995;6:55-73.

7. Levy S, Camm AJ, Saksena S, et al. International consensus on nomenclature and classification of atrial fibrillation: a collaborative project of the Working Group on Arrhythmias and the Working Group of Cardiac Pacing of the European Society of Cardiology and the North American Society of Pacing and Electrophysiology. J Cardiovasc Electrophysiol. 2003;14:443-5.

8. Cox JL. Atrial fibrillation I: a new classification system. $J$ Thorac Cardiovasc Surg. 2003;126:1686-92.

9. Schotten U, Greiser M, Benke D, et al. Atrial fibrillation-induced atrial contractile dysfunction: a tachycardiomyopathy of a different sort. Cardiovasc Res. 2002;53:192-201. 
10. Grogan M, Smith HC, Gersh BJ, Wood DL. Left ventricular dysfunction due to atrial fibrillation in patients initially believed to have idiopathic dilated cardiomyopathy. Am J Cardiol. 1992;69:1570-3.

11. Wang TJ, Larson MG, Levy D, et al. Temporal relations of atrial fibrillation and congestive heart failure and their joint influence on mortality: the Framingham Heart Study. Circulation. 2003; 107:2920-5.

12. Humphries KH, Jackevicius C, Gong Y, et al. Population rates of hospitalization for atrial fibrillation/flutter in Canada. Can J Cardiol. 2004;20:869-76.

13. Stewart S, Hart CL, Hole DJ, McMurray JJ. Population prevalence, incidence, and predictors of atrial fibrillation in the Renfrew/Paisley study. Heart. 2001;86:516-21.

14. Geraci SA, Haan CK. Effect of beta blockers after coronary artery bypass in postinfarct patients: what can we learn from available literature? Ann Thorac Surg. 2002;74:1727-32.

15. Effect of enalapril on survival in patients with reduced left ventricular ejection fractions and congestive heart failure. The SOLVD Investigators. N Engl J Med. 1991;325:293-302.
16. Ferguson TB Jr, Coombs LP, Peterson ED. Preoperative beta-blocker use and mortality and morbidity following CABG surgery in North America. JAMA. 2002;287:2221-7.

17. Gajewski J, Singer RB. Mortality in an insured population with atrial fibrillation. JAMA. 1981;245:1540-4.

18. Franz MR, Karasik PL, Li C, Moubarak J, Chavez M. Electrical remodeling of the human atrium: similar effects in patients with chronic atrial fibrillation and atrial flutter. J Am Coll Cardiol. 1997; 30:1785-92.

19. Allessie MA. Atrial fibrillation-induced electrical remodeling in humans: what is the next step? Cardiovasc Res. 1999;44:10-2.

20. Chua YL, Schaff HV, Orszulak TA, Morris JJ. Outcome of mitral valve repair in patients with preoperative atrial fibrillation. Should the maze procedure be combined with mitral valvuloplasty? J Thorac Cardiovasc Surg. 1994;107:408-15.

21. Savelieva I, Camm AJ. Clinical relevance of silent atrial fibrillation: prevalence, prognosis, quality of life, and management. J Interv Card Electrophysiol. 2000;4:369-82.

\section{Notice of Correction}

Shigemura N, Akashi A, Funaki S, Nakagiri T, Inoue M, Sawabata N, et al. Long-term outcomes after a variety of video-assisted thoracoscopic lobectomy approaches for clinical stage IA lung cancer: A multi-institutional study. J Thorac Cardiovasc Surg. 2006;132:507-12.

The authors report the following error in data analysis: Figure 3 shows the postoperative physical activity of the patients using an accelerometer to quantify their recovery from surgery. The data pertaining to all patients in one of the participant hospitals who received the a-VATS approach are inaccurate. All a-VATS results have been removed from the revised Figure 3. As a result, the authors can no longer conclude that a significant difference exists in the recovery times between the a-VATS and c-VATS groups. The significant difference in the recovery times between the c-VATS and open thoracotomy groups remains valid. All other results from the study remain accurate.

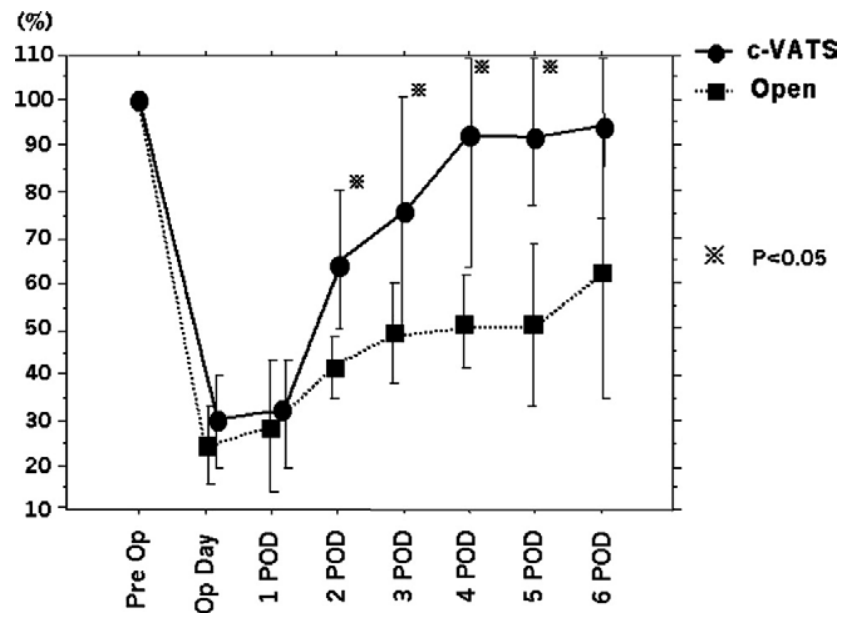

Revised Figure 3. Comparison of the postoperative physical ability as determined by Active Tracer and expressed as the percentage of the preoperative 24-hour value. Time points included are before surgery and $0,1,2,3,4,5$, and 6 days postoperatively. Each value represents the mean \pm standard error at each time point. ${ }^{*} \boldsymbol{P}<.05$ for complete VATS (c-VATS) versus open thoracotomy on postoperative days 2 to 5 . 